\title{
Functional Data Visualization
}

\section{Marc G. Genton ${ }^{\mathrm{a} *}$ and Ying Sun ${ }^{\mathrm{a}}$}

Keywords: Functional boxplot; Functional data; Functional depth; Functional outlyingness; Image data; Magnitude-shape plot; Multivariate functional data; Surface boxplot; Surface data; Trajectory functional boxplot; Two-stage functional boxplot; Visualization.

\begin{abstract}
This article reviews tools to visualize functional data, that is, curves, surfaces/images, and trajectories. These tools are based on ranking functional data by means of notions of depth/outlyingness and make use of methods for functional outlier detections. For univariate functional data, the functional boxplot and surface boxplot are emphasized. For multivariate functional data, the magnitude-shape plot, the two-stage functional boxplot, and the trajectory functional boxplot are described. A bivariate functional dataset of the angles formed by the hip and knee of 39 children over their gait cycles is used throughout for illustration of the various visualization tools. Copyright (C) 2020 John Wiley \& Sons, Ltd.
\end{abstract}

\section{Introduction}

Data in many fields are collected through a process naturally recorded as functional, such as curves, surfaces/images, and trajectories. Figure 1 shows one example of functional data introduced and discussed by Ramsay \& Silverman (2005). The data consist of the angles formed by the hip and knee of 39 children over their gait cycles. The cycle begins and ends when the heel under observation strikes the ground. Each curve is one functional observation measured from each child's gait cycle, and the time is translated into values in $[0,1]$, proportional to the gait cycle. For such datasets, functional data analysis (FDA) provides a considerable number of methodologies and techniques, and has become a popular branch of statistics. A broad overview of FDA and data examples can be found in Ramsay \& Silverman (2005). A more recent review of FDA and discussions can be found in Wang et al. (2016) and references therein.

Visualizing functional data can be a challenge. Genton et al. (2015) discussed and promoted dynamic visualization, coined visuanimation, including functional data visualization. Recently, Castruccio et al. (2019) presented a suite of apps for spatio-temporal data visualization using stereoscopic view and virtual reality on smartphones. In statistics, exploratory data analysis, as the first step of data analysis, requires informative visualization tools. Classical visualization tools designed for scalar data may not be appropriate for functional data. For example, the well-known boxplot is a graphical tool to summarize the distribution of a scalar random variable. Its construction is based on the ranks of the observations. However, for functional data, there is no unique ordering. Data depth is a key idea to generalize ranks to the functional setting. The depth value for each observation measures how central an observation is with respect to

\footnotetext{
a Statistics Program, King Abdullah University of Science and Technology, Thuwal 23955-6900, Saudi Arabia This research was supported by the King Abdullah University of Science and Technology (KAUST).

*Email: marc.genton@kaust.edu.sa
} 

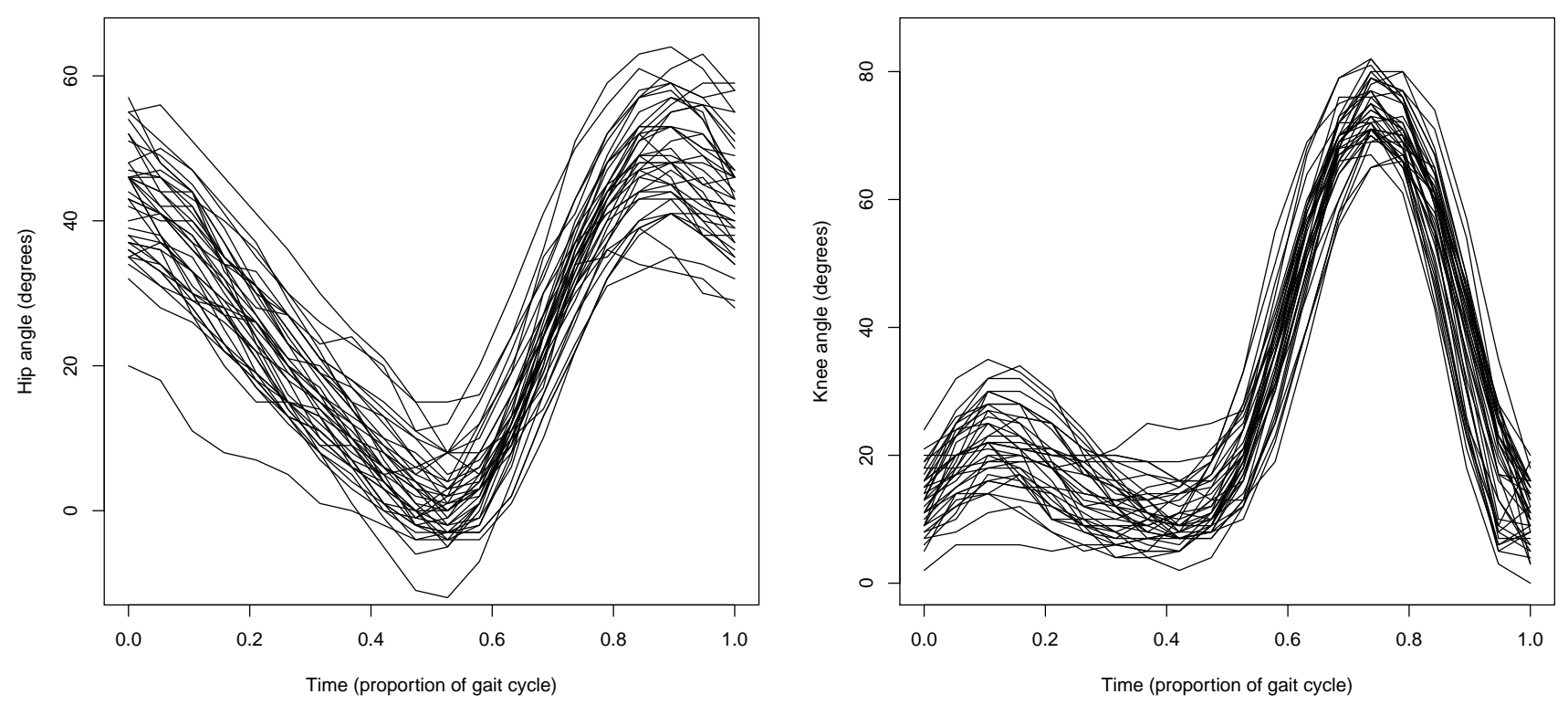

Figure 1. Functional data: The hip (left panel) and knee (right panel) angles of each of the 39 children as they go through their gait cycles. The time interval $[0,1]$ represents a single cycle.

the entire sample. The modified band depth proposed by López-Pintado \& Romo (2009) is one of the most popular depth notions for functional data due to its intuitive geometric interpretation. Other ways for ordering functional data exist, such as the tilting approach introduced by Genton \& Hall (2016). With a given ranking, Sun \& Genton (2011) proposed the functional boxplot as a strong analog to the classical boxplot.

Functional data are often multivariate. For example, Figure 1 shows the hip and knee angles separately, and it does not indicate how the hip angle is paired with the knee angle from the same child. To be able to generalize the boxplot to the multivariate functional setting, multivariate depth is needed. leva \& Paganoni (2013), Claeskens et al. (2014), López-Pintado et al. (2014) and Dai \& Genton (2019) have proposed various methodologies for multivariate functional data ranking.

Rank-based methods are robust against outliers and can be used for outlier detection purposes. The classical boxplot detects potential outliers by the 1.5 times IQR (inter-quartile range) empirical rule. For functional data, this empirical rule was generalized by Sun \& Genton (2011). However, outlier detection is much more complicated for functional data because the observations can be outlying either in magnitude or in shape. Huang \& Sun (2019a) proposed the total variation depth and its decomposition to visualize both magnitude and shape outliers. They compared the outlier detection performance with several existing methods, including the functional boxplot with different depth notions for ranking, such as the modified band depth (López-Pintado \& Romo, 2009) and the extremal depth (Narisetty \& Nair. 2016), the outliergram (Arribas-Gil \& Romo, 2014), the functional outlier map (FOM) with the adjusted outlyingness proposed by Hubert et al. (2015), and the extended FOM with the "directional outlyingness" proposed by Rousseeuw et al. (2018). Recently, Harris et al. (2019) proposed a notion of elastic depth to improve shape outlier detection. 
In Section 2, we describe tools for univariate functional data visualization, whereas in Section 3 we focus on tools for multivariate functional data visualization. We end with some discussions in Section 4.

\section{Univariate Functional Data Visualization}

\subsection{Functional Boxplots}

The functional boxplot first proposed by Sun \& Genton (2011) has been proven a valuable visualization tool for exploratory functional data analysis. Inspired by the classical boxplot introduced as the "Box-and-Whisker plot" by Tukey (1977), the functional boxplot uses robust summary statistics to visualize the distribution of any given functional dataset. Instead of the original "Five Number Summary" in a classical boxplot, i.e., the sample minimum, the first quartile, the median, the third quartile, and the sample maximum, the functional boxplot displays the functional median, the envelope of the $50 \%$ central region and the maximum envelope.

To create a functional boxplot, the first step is to assign ranks to functional observations. Unlike for univariate scalar observations, the ranking for functional data is not unique. The functional boxplot by default uses the ranks induced by the modified band depth (López-Pintado \& Romo, 2009), while the depth values calculated using other depth notions can be provided instead. Denote the order statistics by $y_{[1]}(t), \ldots, y_{[n]}(t)$ according to decreasing depth values. Since the ordering is from the center outwards, the first order statistics, $y_{[1]}(t)$ or the functional observation with the largest depth value, has the most central position and thus called the functional median. The sample 50\% central region is then defined as $C_{0.5}=\left\{(t, y(t)): \min _{r=1, \ldots,\lfloor n / 2\rfloor} y_{[r]}(t) \leq y(t) \leq \max _{r=1, \ldots,\lfloor n / 2\rfloor} y_{[r]}(t)\right\}$, where $\lfloor n / 2\rfloor$ is the smallest integer not less than $n / 2$. The $50 \%$ central region indicates the variability of the central $50 \%$ of the data, and its border is called the envelope representing the box as in the classical boxplot. The functional median is displayed in the central region, which presents the centrality of the data. It is worth to point out that the functional median is one of the observations (or the average of the deepest curves if not unique). In contrast, the envelope of the $50 \%$ central region consists of pieces from different observations and is not an original observation anymore. The maximum envelope can be constructed in a similar way and is the analog to the whiskers in the classical boxplot.

In the functional boxplot, the maximum envelope is constructed after removing outliers by the 1.5 times the $50 \%$ central region empirical rule, where the fences are determined by inflating the envelope of the $50 \%$ central region by 1.5 times its range for each $t$. The constant factor $F$ is set to be 1.5 by default as in the 1.5 times IQR rule in the classical boxplot. However, it can be modified by users. For instance, Sun \& Genton (2012a) proposed an adjusted functional boxplot for outlier detection in spatio-temporal data, where the factor $F$ is adjusted according to the dependence among the functional observations.

To illustrate the construction of the functional plot, we consider the gait data, as shown in Figure 1. Figure 2 shows the functional boxplots of hip and knee angles for two choices of $F$ for the illustration of outlier detection. Compared to the plots of original curves, the functional median curves for the hip and knee angles show more clearly the different phases of the data. For example, we see that at the beginning, the hip angle decreases from its maximum angle to its minimum near zero, while the knee angle first increases from zero to a local maximum near zero and then decreases to near zero. When the hip angle switches to a phase of increase, the knee angle increases sharply to its maximum as the leg prepares to leave the floor. During the last phase, the knee angle decreases rapidly to zero as the leg extends, and the hip angle levels off. The magenta area boxes the 50\% central regions with the corresponding envelopes displayed in blue, which indicate the variability of these gait cycles among the 39 children. When $F=1.5$, there were no outliers in both functional boxplots. For the hip angle data, one outlier is detected when decreasing $F$ to 1.2 , and for the knee angle data, when $F=0.61$, one outlier is identified. These two outliers in red show slightly different features from the 
$F=1.5$

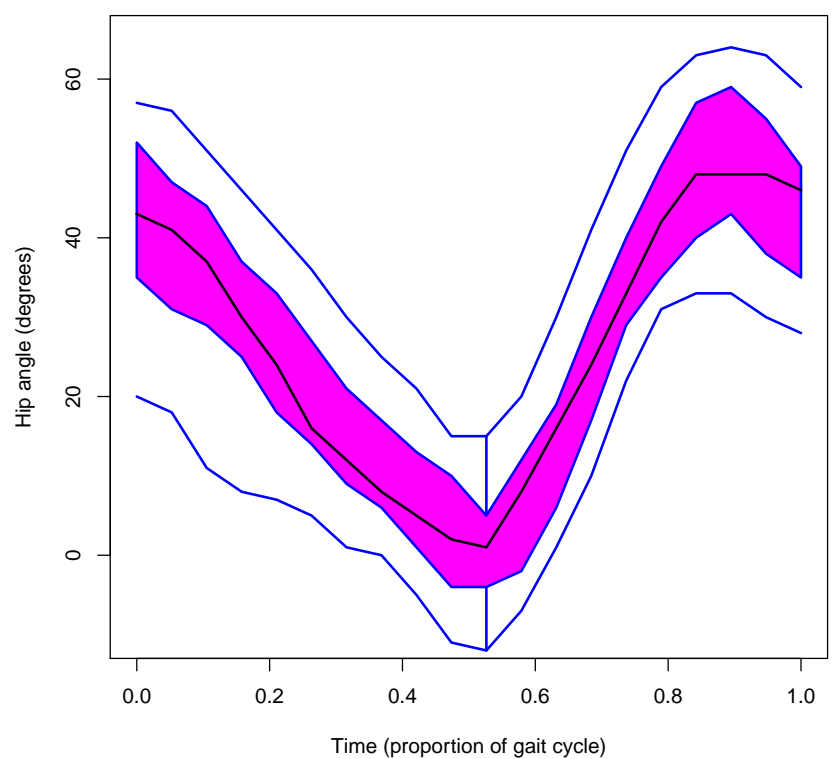

$F=1.2$

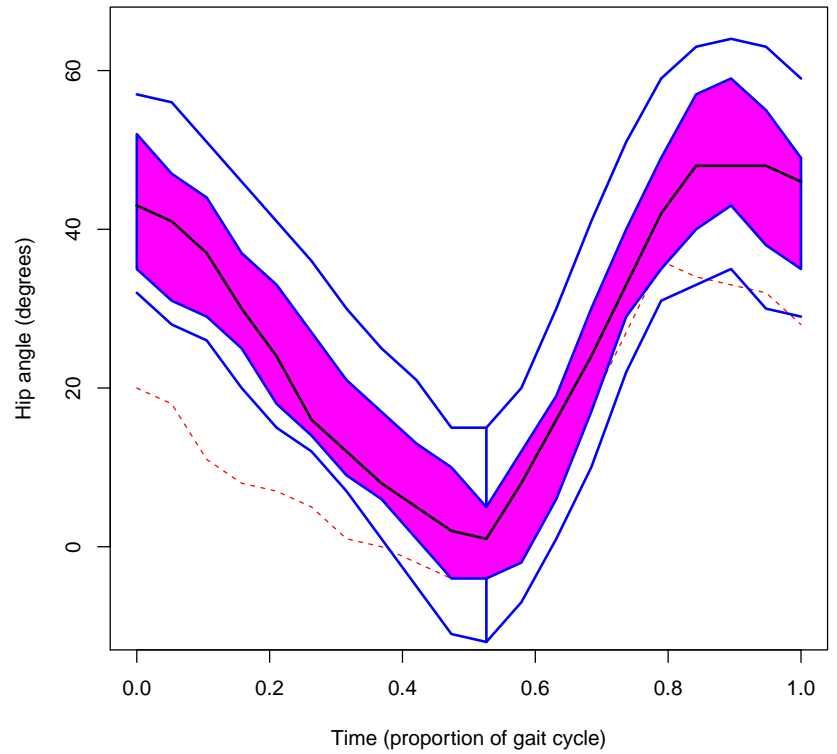

$F=1.5$

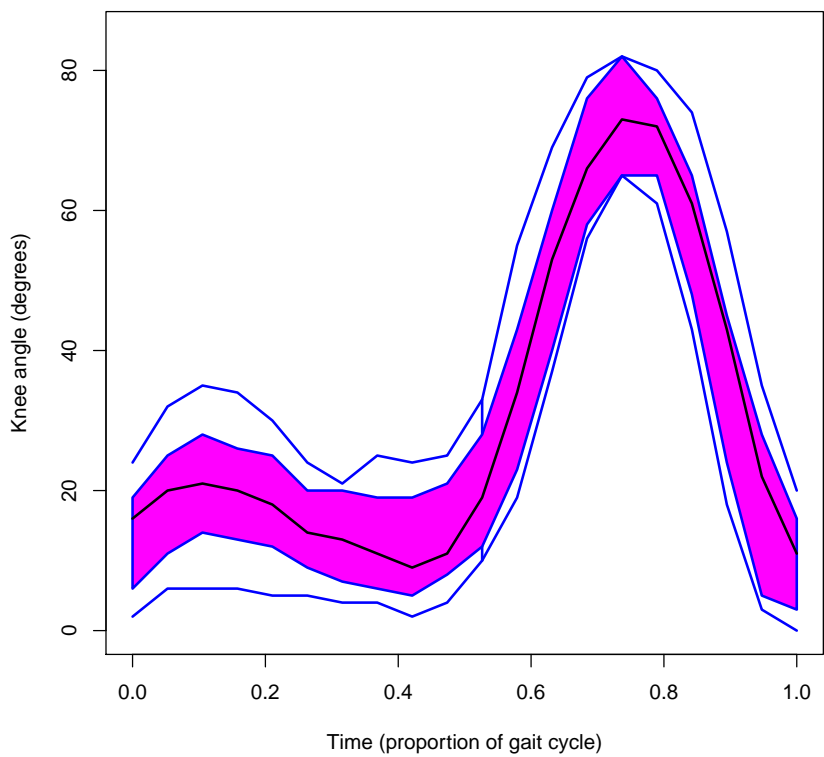

$F=0.61$

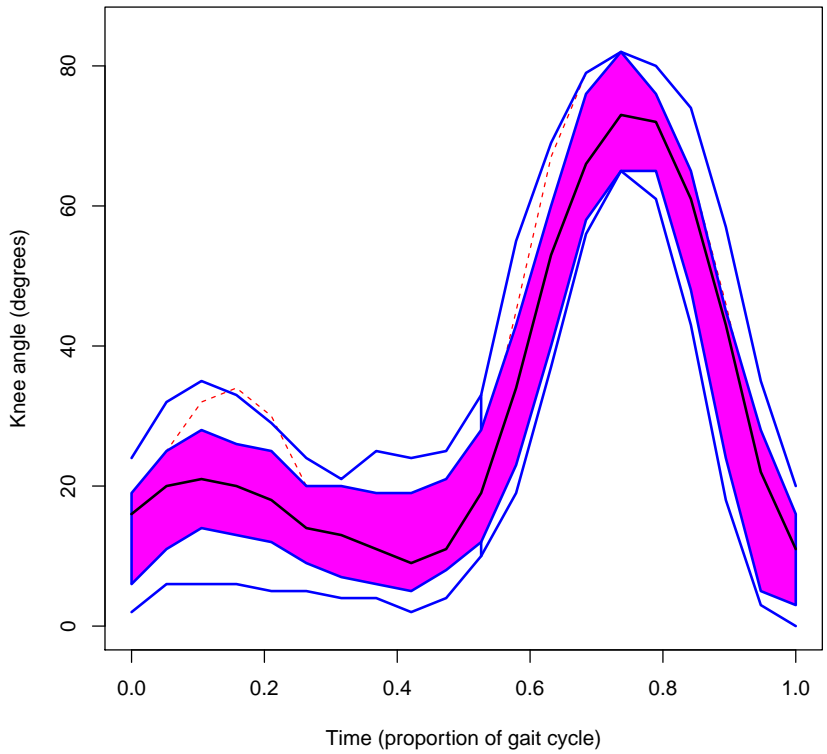

Figure 2. The functional boxplots for the hip and knee angles of each of the 39 children. The black curve in each functional boxplot is the median, the magenta box is the $50 \%$ central region and the red dashed curves are the potential outliers identified by the $F$ times the $50 \%$ central region rule. 
majority of the curves. Finally, the maximum envelopes in blue present the range of the non-outlying observations. As pointed out by Sun \& Genton (2012a), the adjustment of $F$ is not so crucial for visualization purposes. However, it needs to be carefully selected for outlier detection. More discussion on the selection of the adjustement factor $F$ and various examples can be found in Sun \& Genton (2012a).

Thanks to the fast algorithm developed in Sun et al. (2012), the functional boxplot has been widely used and applied to various types of functional datasets, from visualization, outlier detection to simulation, and model diagnostics. For instance, the functional boxplot was used to detect outliers in functional autoregressive models by Martínez-Hernández et al. (2019); Huang \& Sun (2019b) proposed to assess spatio-temporal covariance properties using functional boxplots; Sun \& Genton (2012b) first used the functional boxplot to summarize simulation results of robust functional median polish for univariate ANOVA and later it was extended to MANOVA by Qu et al. (2020). It has also been employed to analyze and visualize the log periodograms of EEG time series data in the spectral domain (Ngo et al., 2015), and Euan \& Sun (2019) used functional boxplots to visualize clustering results for time series data and proposed the directional functional boxplot for visualizing the directional spectrum. Sun \& Stein (2015) utilized the functional boxplot to assess the statistical properties of their spatio-temporal rainfall model, and La Vecchia \& Ronchetti (2019) made use of the functional boxplot to examine the variability of the PP-plot for comparing different methods when approximating the distribution of estimators and test statistics.

Other visualization tools exist for functional data, such as the functional bagplot and the functional highest density region boxplot (Hyndman \& Shang (2010)) based on the first two robust principal component scores, and boxplots for amplitude, phase and vertical translation (Xie et al. 2017) in the context of functional data registration.

\subsection{Surface Boxplots}

Sun \& Genton (2011) presented an illustration of the surface boxplot as a potential extension of the functional boxplot when observations are surfaces or images. The surface boxplot can be constructed similarly to the functional boxplot once the ranking of the sample surfaces is obtained. However, the 3D visualization is not trivial. A user-friendly visualization tool was developed by Genton et al. (2014) and illustrated on several real-world data examples. Yan et al. (2020) utilized this tool to visualize estimated covariance matrices from simulations by treating the matrices as images.

\section{Multivariate Functional Data Visualization}

In the gait cycle data example, the knee and hip angles of each child must be dependent and can be visualized jointly as multivariate functional data, i.e., a random vector indexed by time. For multivariate functional data, the outlier detection is even more complicated because multivariate functional outliers are not necessarily marginal outliers and the outlyingness could also occur in magnitude, shape or both, as in the univariate case. There is no clear definitition for each type of outliers. Dai et al. (2020) proposed a set of transformation operations on functional data for outlier detection purposes, where potential outliers are either magnitude or shape outliers after suitable transformations. Next, we describe several tools for the visualization of multivariate functional data as well as their outlier detection.

\subsection{Magnitude-Shape Plots}

In order to rank multivariate functional data, Dai \& Genton (2019) introduced the notion of directional outlyingness, a vector $\mathbf{O}(t)$ which measures at time $t$ the centrality of functional data by assessing the level and the direction of their deviation from the central region together. The mean vector $\mathbf{M O}$ and scalar variance $\mathrm{VO}$ of $\mathbf{O}(t)$ over time were defined to quantify the magnitutde outlyingness and the shape outlyingness, respectively, of curves. Dai 
\& Genton (2018b) proposed a new graphical tool, the magnitude-shape (MS) plot of (MO,VO), for visualizing both the magnitude and shape outlyingness of multivariate functional data. They provided also a dividing elliptical curve (or ellipsoidal surface) to separate non-outlying data from the outliers. They demonstrated through Monte Carlo simulations and data applications that the MS-plot is superior to existing tools for visualizing centrality and detecting outliers for functional data, such as the functional outlier map (FOM) of Hubert et al. (2015) and Rousseeuw et al. (2018), both of which fail to detect shape outliers. Dai \& Genton (2018b) also proposed the MS-plot array which displays marginal MS-plots on the diagonal and pairwise bivariate MS-plots on the off-diagonals.

Figure 3 depicts the bivariate and marginal data of hip and knee angles of each of the 39 children, as well as the associated bivariate and marginal MS-plots. The light-blue ellipsoid and ellipses represent the thresholds to flag

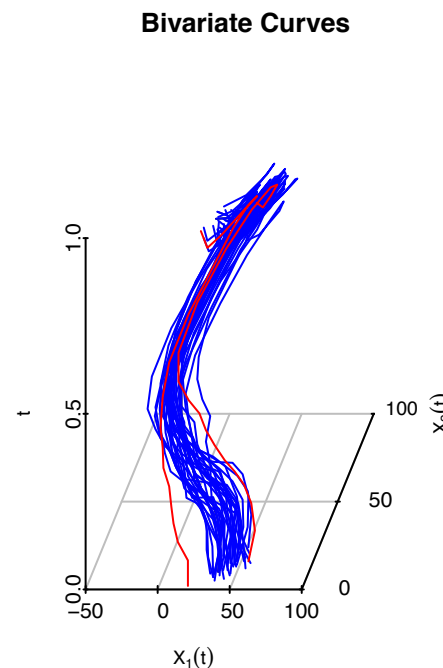

Bivariate MS-Plot

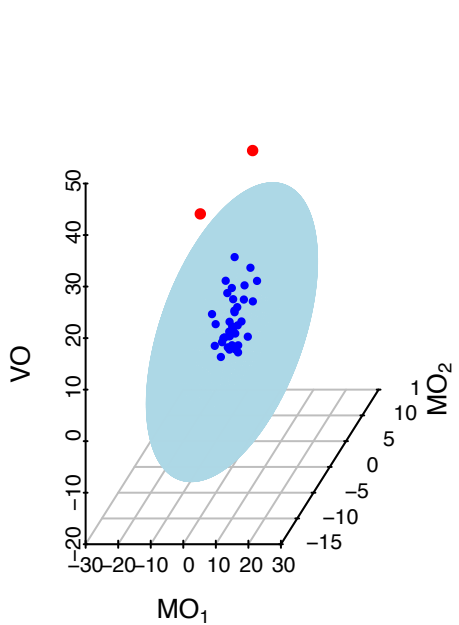

Hip angle

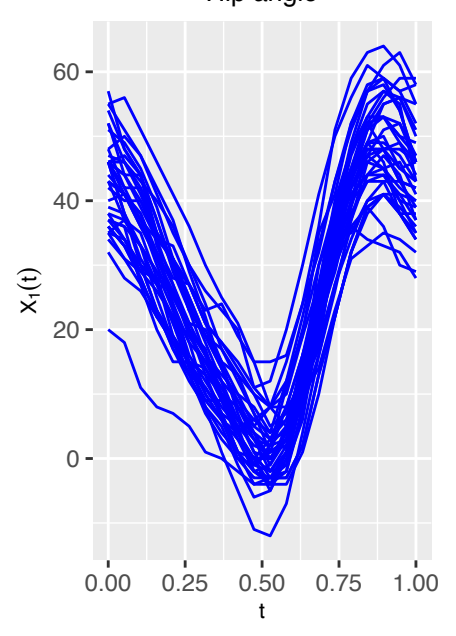

MS-Plot

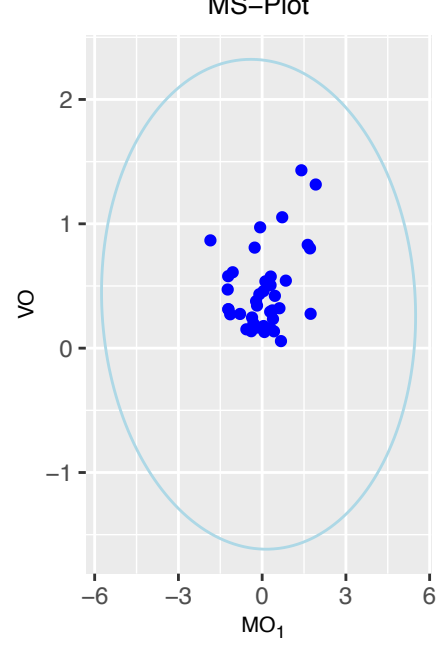

Knee angle

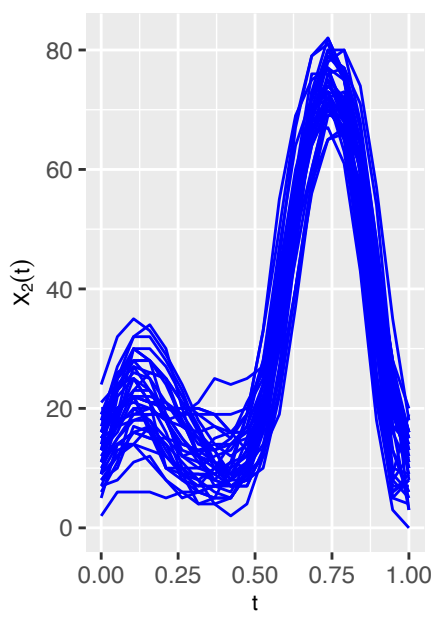

MS-Plot

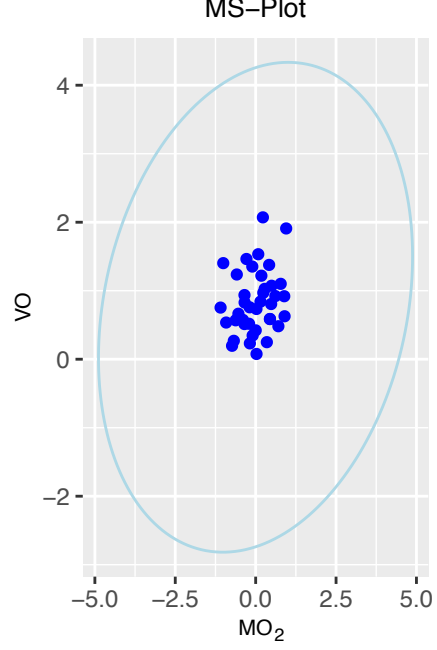

Figure 3. The bivariate and marginal MS-plots for the hip and knee angles of each of the 39 children. The light-blue ellipsoid and ellipses represent the thresholds to flag potential outliers. The red curves/points are the potential outliers identified. They are joint outliers but not marginal outliers. 
potential outliers. The red curves/points are the potential outliers identified. They are joint outliers but not marginal outliers. Hence, it is important to apply joint rather than marginal methods in the multivariate case.

\subsection{Two-Stage Functional Boxplots}

Dai \& Genton (2018a) proposed a two-stage functional boxplot for the visualization and exploratory functional data analysis of multivariate curves. They showed that the shape of the original functional boxplot is sensitive to shape outliers and proposed to combine it with an outlier-detection procedure based on the aforementioned functional directional outlyingness in order to account for both the magnitude and shape outlyingness of functional data. In a first stage, shape outliers are identified, and in a second stage, the traditional functional boxplot is constructed on the remaining non-shape-outlying curves. This combination is robust to various types of outliers and allows to more precisely capture the data structures than does the functional boxplot alone. Moreover, it can provide both marginal and joint analyses of the multivariate curves.

We apply the two-stage functional boxplot to the the hip and knee angles of each of the 39 children in Figure 4. The green curves are the potential shape outliers identified in the first stage. The shapes of the traditional functional boxplots in the second stage become slightly different from the shapes seen in the first row of Figure 2 in particular for the $50 \%$ central regions in magenta and the whiskers.

Two-stage Functional Boxplot

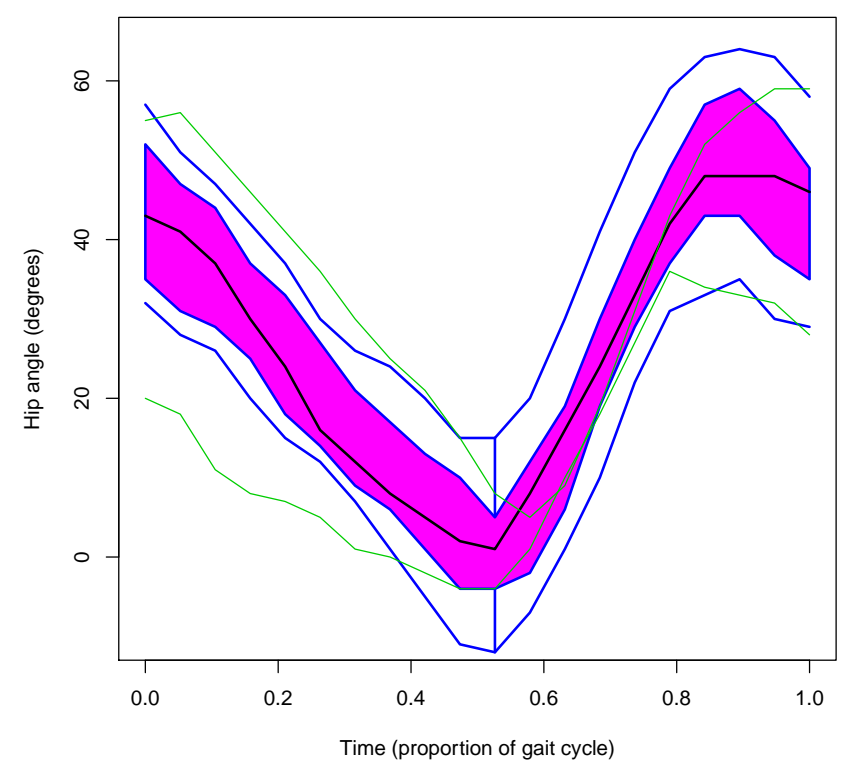

Two-stage Functional Boxplot

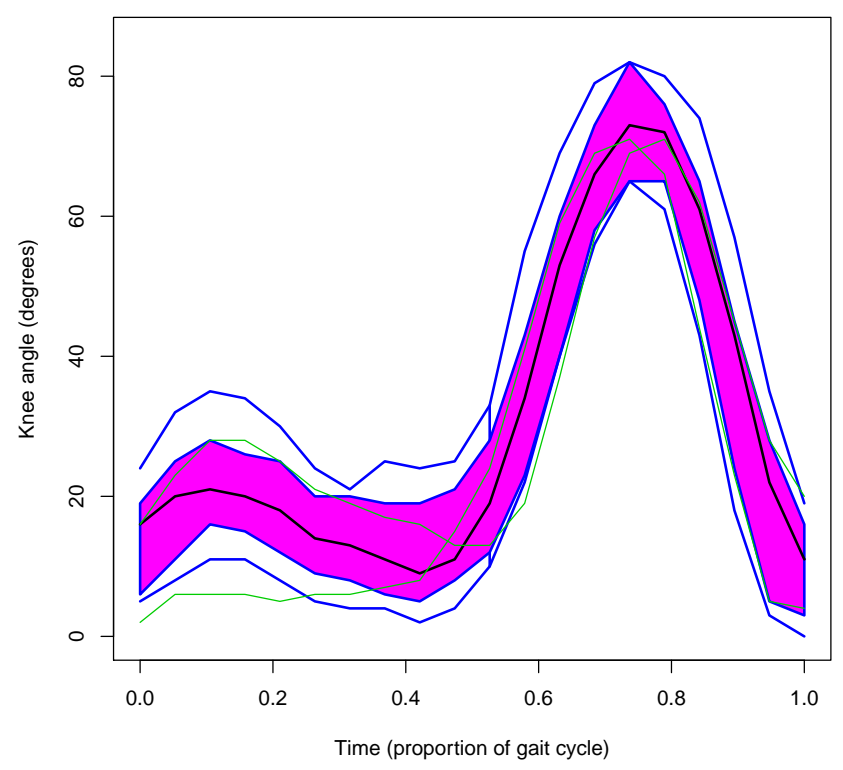

Figure 4. The two-stage functional boxplots for the hip (left panel) and knee (right panel) angles of each of the 39 children. The green curves are the potential bivariate shape outliers identified.

\subsection{Trajectory Functional Boxplots}

An important subset of multivariate functional data consists of the so-called trajectory functional data, which are viewed without a dimension/axis for time. Examples of trajectory functional data include the paths of hurricanes or 
migrating birds in the 2D plane. One could also view the gait data in the hip/knee 2D plane, see left panel of Figure 5 . Note that such data could be open or closed curves.

Mirzargar et al. (2014) introduced the curve boxplot as a generalization of the boxplot to an ensemble of curves. Yao et al. (2019) proposed two exploratory tools for trajectory functional data: the trajectory functional boxplot and the modified simplicial band depth (MSBD) of López-Pintado et al. (2014) versus wiggliness of directional outlyingness (WO) plot. They allow to visualize the centrality of trajectory functional data. Yao et al. (2019) defined the WO index, a measure of the roughness of curves, to effectively measure the shape variation of curves and to detect shape outliers. In addition, MSBD provides a center-outward ranking and identifies magnitude outliers. Using the measures of MSBD and WO, the functional boxplot of the trajectories reveals center-outward patterns and potential outliers using the raw curves. Moreover, the MSBD-WO plot illustrates such patterns and outliers in a space spanned by MSBD and WO.

Figure 5 depicts the trajectory functional boxplot and the MSBD-WO plot for the 2D trajectories of hip and knee angles of each of the 39 children. The black curve/point represents the median. The purple/magenta/pink curves/points represent the $25 \% / 50 \% / 75 \%$ central regions. The three red dashed curves/points are the potential
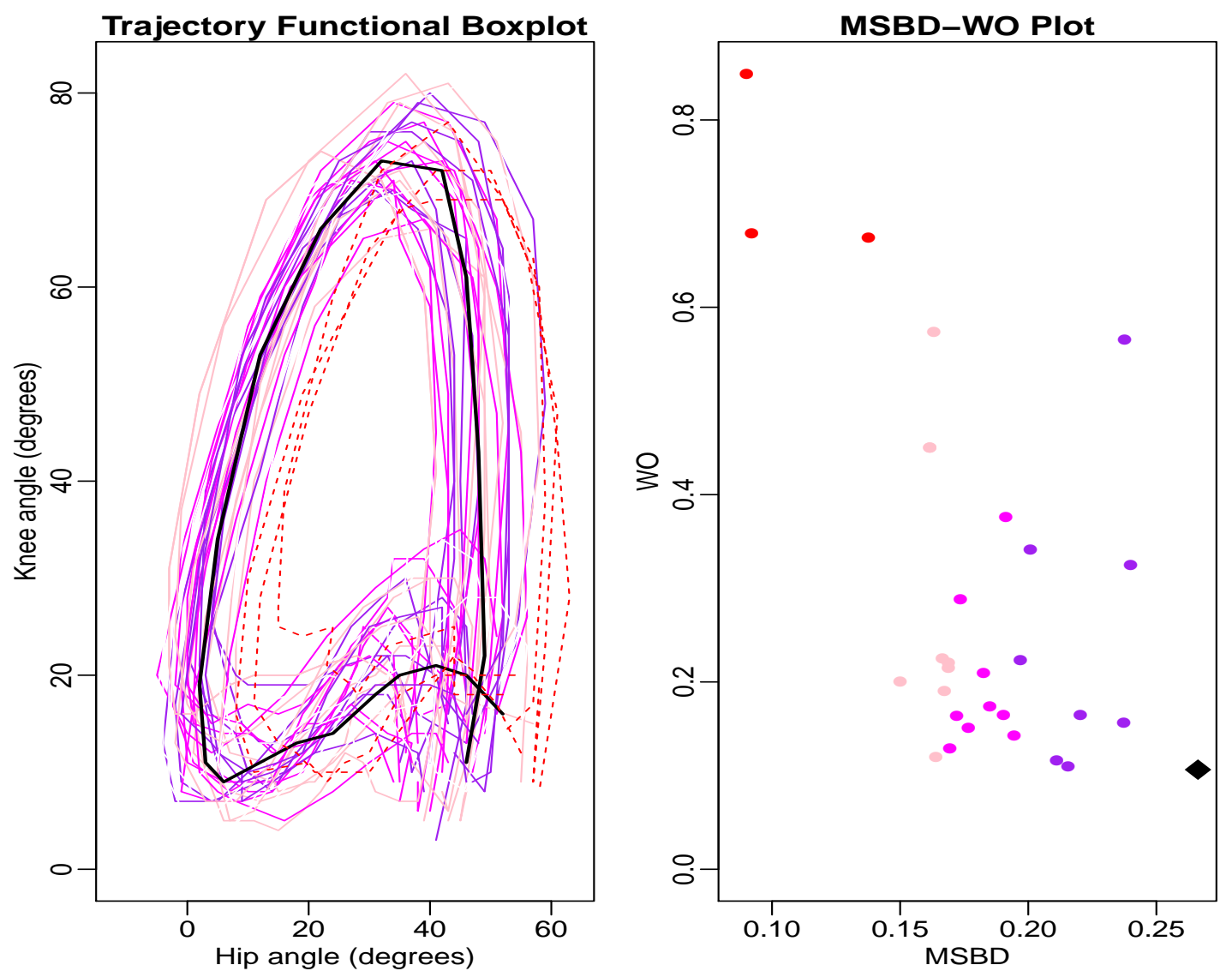

Figure 5. The trajectory functional boxplot (left) and the MSBD-WO plot (right) for the bivariate trajectories of hip and knee angles of each of the 39 children. The black curve/point represents the median. The purple/magenta/pink curves/points represent the $25 \% / 50 \% / 75 \%$ central regions. The three red dashed curves/points are the potential outliers identified. 
outliers identified. Note that the outliers detected with WO are not necessarily the same as those detected with directional outlyingness. Yao et al. (2019) showed that WO is a more effective measure for detecting outliers in trajectory functional data.

\section{Conclusions}

The visualization of functional data is essential for many applications. This article focused on visualization tools and outlier detection techniques based on functional data ranking, from univariate to multivariate cases. The reviewed methods have been illustrated on a dataset consisting of the angles formed by the hip and knee recorded from 39 children over their gait cycles.

These rank-based methods are robust and do not require any model or distribution assumptions. However, the computational cost might limit their capability in practice. The functional boxplot is implemented by the user-friendly fbplot command available in the fda packages (Ramsay et al., 2018) in R (R Core Team, 2019) and in MATLAB (MATLAB, 2018). It is straightforward to extract important statistics from the fbplot command for future analysis and visualization purposes. Although it is possible to use depth values from other notions to rank functional data, the functional boxplot by default uses the fast algorithm of Sun et al. (2012) for calculating the modified band depth values which makes many applications feasible.

We have only illustrated univariate and bivariate curves, as well as trajectories, in this article. With modern data collection instruments and various applications from different research fields, the observations can be even more complex functional objects. To understand features of complicated data from all the aspects, visualization is particularly useful for exploratory functional data analysis, yet challenging. There are many open problems related to robust statistics and computations that require further research. Moreover, we have only focused on discussing the visualization of functional observations. Many core methods in FDA have common graphics to visualize the results from standard analyses, such as scree plots for functional principal component analysis and coefficient function plots in regression models. Wrobel et al. (2016) illustrated how to create interactive graphics for functional data analyses with the refund.shiny package (Wrobel \& Goldsmith, 2016) in R.

\section{Related Articles}

\section{Boxplot; Data Visualization; Exploratory Data Analysis; Functional Data Analysis.}

\section{References}

Arribas-Gil, A \& Romo, J (2014), 'Shape outlier detection and visualization for functionaldata: the outliergram.' Biostatistics, 15(4), pp. 603-619.

Castruccio, S, Genton, MG \& Sun, Y (2019), 'Visualizing spatiotemporal models with virtual reality: from fully immersive environments to applications in stereoscopic view,' J. Roy. Statist. Soc. Ser. A, 182(2), pp. 379-387.

Claeskens, G, Hubert, M, Slaets, L \& Vakili, K (2014), 'Multivariate functional halfspace depth,' J. Amer. Statist. Assoc., 109(505), pp. 411-423.

Dai, W \& Genton, MG (2018a), 'Functional boxplots for multivariate curves,' Stat, 7, p. e190.

Dai, W \& Genton, MG (2018b), 'Multivariate functional data visualization and outlier detection,' J. Comput. Graph. Statist., 27(4), pp. 923-934. 
Dai, W \& Genton, MG (2019), 'Directional outlyingness for multivariate functional data,' Comput. Statist. Data Anal., 131, pp. 50-65.

Dai, W, Mrkvička, T, Sun, Y \& Genton, MG (2020), 'Functional outlier detection and taxonomy by sequential transformations,' Comput. Statist. Data Anal., p. to appear.

Euan, C \& Sun, Y (2019), 'Directional spectra-based clustering for visualizing patterns of ocean waves and winds,' J. Comput. Graph. Statist., 28(3), pp. 659-670.

Genton, MG, Castruccio, S, Crippa, P, Dutta, S, Huser, R, Sun, Y \& Vettori, S (2015), 'Visuanimation in statistics,' Stat, 4, pp. 81-96.

Genton, MG \& Hall, P (2016), 'A tilting approach to ranking influence,' J. R. Stat. Soc. Ser. B. Stat. Methodol., 78(1), pp. 77-97.

Genton, MG, Johnson, C, Potter, K, Stenchikov, G \& Sun, Y (2014), 'Surface boxplots,' Stat, 3, pp. 1-11.

Harris, T, Tucker, JD, Li, B \& Shand, L (2019), 'Elastic depths for detecting shape anomalies in functional data,' arXiv:1907.06759.

Huang, H \& Sun, Y (2019a), 'A decomposition of total variation depth for understanding functional outliers,' Technometrics, 61(4), pp. 445-458.

Huang, H \& Sun, Y (2019b), 'Visualization and assessment of spatio-temporal covariance properties,' Spat. Statist., 34, p. 100272.

Hubert, M, Rousseeuw, PJ \& Segaert, P (2015), 'Multivariate functional outlier detection,' Stat. Methods Appl., 24(2), pp. 177-202.

Hyndman, RJ \& Shang, HL (2010), 'Rainbow plots, bagplots, and boxplots for functional data,' J. Comput. Graph. Statist., 19(1), pp. 29-45.

leva, F \& Paganoni, AM (2013), 'Depth measures for multivariate functional data,' Comm. Statist. Theory Methods, 42(7), pp. 1265-1276.

La Vecchia, D \& Ronchetti, E (2019), 'Saddlepoint approximations for short and long memory time series: a frequency domain approach,' J. Econometrics, 213(2), pp. 578-592.

López-Pintado, S \& Romo, J (2009), 'On the concept of depth for functional data,' J. Amer. Statist. Assoc., 104(486), pp. $718-734$.

López-Pintado, S, Sun, Y, Lin, JK \& Genton, MG (2014), 'Simplicial band depth for multivariate functional data,' Adv. Data Anal. Classif., 8(3), pp. 321-338.

Martínez-Hernández, I, Genton, MG \& González-Farías, G (2019), 'Robust depth-based estimation of the functional autoregressive model,' Comput. Statist. Data Anal., 131, pp. 66-79.

MATLAB (2018), version 9.4.0 (R2018a), The MathWorks Inc., Natick, Massachusetts.

Mirzargar, M, Whitaker, RT \& Kirby, RM (2014), 'Curve boxplot: Generalization of boxplot for ensembles of curves,' IEEE Trans. Vis. Comput. Graph., 20(12), pp. 2654-2663.

Narisetty, NN \& Nair, VN (2016), 'Extremal depth for functional data and applications,' J. Amer. Statist. Assoc., 111(516), pp. 1705-1714. 
Ngo, D, Sun, Y, Genton, MG, Wu, J, Srinivasan, R, Cramer, S \& Ombao, H (2015), 'An exploratory data analysis of electroencephalograms using the functional boxplots approach,' Front. Neurosci., 9(282), pp. 1-18.

Qu, Z, Dai, W \& Genton, MG (2020), 'Robust functional multivariate analysis of variance with environmental applications,' Unpublished Manuscript.

R Core Team (2019), R: A Language and Environment for Statistical Computing, R Foundation for Statistical Computing, Vienna, Austria.

Ramsay, JO \& Silverman, BW (2005), Functional Data Analysis, Springer Series in Statistics, Springer, New York, second edn.

Ramsay, JO, Wickham, H, Graves, S \& Hooker, G (2018), fda: Functional Data Analysis, R package version 2.4.8.

Rousseeuw, PJ, Raymaekers, J \& Hubert, M (2018), 'A measure of directional outlyingness with applications to image data and video,' J. Comput. Graph. Statist., 27(2), pp. 345-359.

Sun, Y \& Genton, MG (2011), 'Functional boxplots,' J. Comput. Graph. Statist., 20(2), pp. 316-334.

Sun, Y \& Genton, MG (2012a), 'Adjusted functional boxplots for spatio-temporal data visualization and outlier detection,' Environmetrics, 23(1), pp. 54-64.

Sun, Y \& Genton, MG (2012b), 'Functional median polish,' J. Agric. Biol. Environ. Stat., 17(3), pp. 354-376.

Sun, Y, Genton, MG \& Nychka, DW (2012), 'Exact fast computation of band depth for large functional datasets: How quickly can one million curves be ranked?' Stat, 1, pp. 68-74.

Sun, Y \& Stein, ML (2015), 'A stochastic space-time model for intermittent precipitation occurrences,' Ann. Appl. Stat., 9(4), pp. 2110-2132.

Tukey, JW (1977), Exploratory Data Analysis, Addison-Wesley.

Wang, JL, Chiou, JM \& Müller, HG (2016), 'Functional data analysis,' Annu. Rev. Stat. Appl., 3(1), pp. 257-295.

Wrobel, J \& Goldsmith, J (2016), refund.shiny: Interactive Plotting for Functional Data Analyses, R package version 0.3.0.

Wrobel, J, Park, SY, Staicu, AM \& Goldsmith, J (2016), 'Interactive graphics for functional data analyses,' Stat, 5(1), pp. 108-118.

Xie, W, Kurtek, S, Bharath, K \& Sun, Y (2017), 'A geometric approach to visualization of variability in functional data,' J. Amer. Statist. Assoc., 112(519), pp. 979-993.

Yan, Y, Huang, HC \& Genton, MG (2020), 'Vector autoregressive models with spatially structured coefficients for time series on a spatial grid,' arXiv:2001.02250.

Yao, Z, Dai, W \& Genton, MG (2019), 'Trajectory functional boxplots,' arXiv:1904.10792. 